\title{
Theoretical Analysis of Inertia-like Switching in Magnets: Applications to a Synthetic Antiferromagnet
}

\author{
Satadeep Bhattacharjee, Anders Bergman, Andrea Taroni, Johan Hellsvik, Biplab Sanyal, and Olle Eriksson \\ Department of Physics and Astronomy, Box 516, 75120, Uppsala University, Uppsala, Sweden
}

(Received 3 September 2011; published 29 March 2012)

\begin{abstract}
The magnetization dynamics of a synthetic antiferromagnet subjected to a short-magnetic-field pulse has been studied by using a combination of first principles calculations and atomistic spin-dynamics simulations. We observe switching phenomena on the time scale of tens of picoseconds, and inertia-like behavior in the magnetization dynamics. We explain the latter in terms of a dynamic redistribution of magnetic energy from the applied-field pulse to other possible energy terms, such as the exchange interaction and the magnetic anisotropy, without invoking concepts such as the inertia of an antiferromagnetic vector. We also demonstrate that such dynamics can also be observed in a ferromagnetic material where the incident-field pulse pumps energy to the magnetic anisotropy.
\end{abstract}

DOI: 10.1103/PhysRevX.2.011013

Subject Areas: Computational Physics, Magnetism, Materials Science

\section{INTRODUCTION}

Magnetization reversal by applying an externalmagnetic field [1] is central to magnetic data storage technologies. Magnetic random access memories (RAMs) [2] based on magnetoresistance, in which magnetic bits can be described in terms of high (1) or low (0) resistance units, are among the most promising memory devices for the near future. The magnetoresistance unit consists of a nonmagnetic layer sandwiched between two magnetic layers and, depending on whether the nonmagnetic layer is metallic or insulating, one either refers to giant magnetoresistance $[3,4]$ or to tunnel magnetoresistance $[5,6]$. These memory devices, which are characterized by a very dense architecture and are capable of performing with low power consumption, are proposed as "universal" memory devices, since they carry all the advantages of other devices such as static RAM, dynamic RAM and flash memory [7]. A magnetic RAM capable of performing at the speed of static RAM along with a dense architecture comparable to dynamic RAM, coupled with nonvolatility (i.e., it does not result in information loss when the power is switched off), is, to some extent, limited by its writing speed. This depends on the magnetization reversal time of its constituent magnetic units.

The potential for applications of magnetization dynamics in technology, combined with the new knowledge of fundamental-magnetic interactions in nanosized materials, has caused a large increase in the interest in this field. Several mechanisms for shorter switching speeds have been proposed, e.g., toggle switching [7], all-optical switching [8], switching accelerated by antiferromagnetic

Published by the American Physical Society under the terms of the Creative Commons Attribution 3.0 License. Further distribution of this work must maintain attribution to the author(s) and the published article's title, journal citation, and DOI. exchange [9], and so forth. One recent suggestion is the socalled inertia-driven switching [10]. Here, a short-magnetic-field pulse, optically generated, with a duration of $100 \mathrm{fs}$, was found to cause a dynamic behavior of the magnetism of $\mathrm{HoFeO}_{3}$, which went on for several tens of picoseconds (ps). In Ref. [10], it was pointed out that this behavior is expected only for materials with antiferromagnetic exchange interactions, either for bulk compounds with an intrinsic antiferromagnetic exchange (e.g., like $\mathrm{HoFeO}_{3}$ ) or in artificial-magnetic trilayers or multilayers, with an antiferromagnetic interlayer exchange. It was argued that, for materials with antiferromagnetic exchange interactions, the switching can be described by a secondorder-differential equation of the so-called antiferromagnetic unit vector, and hence has features associated with an inertia. It was, in particular, argued that the inertia accumulated during the short pulse was responsible for the magnetization dynamics that lasted even after the pulse was switched off.

In this paper, we address the microscopic mechanisms behind this kind of switching for a magnetic trilayer, using atomistic spin-dynamics simulations [11,12]. We have chosen for our studies an $\mathrm{Fe} / \mathrm{Cr} / \mathrm{Fe}$-model system, with an antiferromagnetic interlayer-exchange interaction (IEC). A schematic figure of the system considered is shown in Fig. 1. The exchange constants are calculated from first principles by mapping the ground-stateelectronic structure to a Heisenberg Hamiltonian, using the Liechtenstein-Katnelson-Gubanov method [13]. We calculate the time evolution of the magnetizations of an $\mathrm{Fe} / \mathrm{Cr} / \mathrm{Fe}$ trilayer using a spin-dynamics simulation based on the Landau-Lifshitz-Gilbert method, using the UPPASD package [12]. In our simulations, we analyze the different terms that play a role in the switching behavior of materials with an antiferromagnetic exchange, which are exposed to ultra-short pulses. Although our simulations reproduce much of the observations reported in Ref. [10], our interpretation of the microscopic mechanisms is different. 
We argue that a more appropriate description of the mechanism behind this switching is a redistribution of the energy from the ultrafast-magnetic pulse to the antiferromagnetic exchange interaction and magnetic anisotropy of the material. After the pulse is switched off, the dynamics is governed by an effective field, now composed only of the exchange and anisotropy fields, that temporally evolves for much longer time scales than the initial pulse. The dynamically evolving magnetization carries the effect of the field pulse applied at the initial phase of the simulation and the effect persists through the course of simulation.

\section{METHOD}

The temporal evolution of sublattice magnetizations is calculated using the dynamical equation of the LandauLifshitz-Gilbert form expressed in terms of atomic moments $[11,12,14]$,

$\frac{d \mathbf{m}_{\mathbf{i}}(t)}{d t}=-\frac{\gamma}{1+\alpha^{2}} \mathbf{m}_{\mathbf{i}}(t) \times\left\{\mathbf{H}_{\mathbf{e f f}}^{\mathbf{i}}+\frac{\alpha}{m_{s}^{i}}\left[\mathbf{m}_{\mathbf{i}}(t) \times \mathbf{H}_{\text {eff }}^{\mathbf{i}}\right]\right\}$,

where $\mathbf{m}_{\mathbf{i}}(t)$ is the atomic moment on the $\mathbf{i t h}$ site at time $t . \gamma$ is the gyro-magnetic ratio and $\alpha$ is the Gilbert damping factor. $m_{s}^{i}$ is the saturation moment of the atomic moment at the $\mathbf{i t h}$ site. The effective field $\mathbf{H}_{\text {eff }}^{\mathbf{i}}$ on the $\mathbf{i t h}$ atom is calculated from the effective-magnetic Hamiltonian given by

$$
\mathcal{H}_{\mathrm{Mag}}=\mathcal{H}_{\mathrm{ex}}+\mathcal{H}_{\mathrm{MA}}+\mathcal{H}_{a},
$$

where subscript "ex" labels the exchange interaction, the subscript "MA" labels the magnetic anisotropy, and the subscript $a$ labels the applied field. The effective field of atom $\mathbf{i}$ can be calculated through

$$
\mathbf{H}_{\text {eff }}^{\mathbf{i}}=-\frac{\partial \mathcal{H}_{\mathrm{Mag}}}{\partial \mathbf{m}_{\mathbf{i}}(t)} .
$$

As one can see, the effective field defined above is entirely determined by the instantaneous-magnetic configuration and there is no dissipation of magnetic energy through any other degrees of freedom, such as itinerant electrons. The time nonlocalities of the effective field due to the coupling to other degrees of freedom are discussed in Ref. [15].

The equations for atomistic spin dynamics, discussed above, are relevant for magnetization processes that take place on a time scale down to subpicoseconds, since they rely on a Born-Oppenheimer-like approximation for the slower moving atomic spins, as compared to the considerably faster motion of the electrons [11]. This approximation is expected to apply to phenomena faster than the subpicosecond regime, and is hence relevant for the present study. For even faster phenomena, alternative descriptions must be considered, possibly based on time-dependent density-functional theory [16] in combination with the
Liouville equation for the time evolution of the density matrix [17].

The first term in the Hamiltonian in Eq. (2) describes the interatomic exchange interaction between all atoms of the simulation cell. It can be separated into two parts: (i) a pair exchange between the atomic moments in the same layer (intralayer exchange coupling) and (ii) the exchange coupling between the moments residing in different layers (interlayer-exchange coupling). In our simulations, the exchange interactions are taken into account by a mapping of the system to a classical Heisenberg Hamiltonian,

$$
\mathcal{H}=-\frac{1}{2} \sum_{i \neq j} J_{i j} \mathbf{m}_{i} \cdot \mathbf{m}_{j},
$$

between pairs of magnetic moments $\mathbf{m}_{i}$ and $\mathbf{m}_{j}$ that are coupled through the exchange interactions $J_{i j}$ calculated from first principles. More details of the calculation are provided in Refs. [9,13]. The second term of Eq. (2) represents the magnetic anisotropy. The form of the magnetic-anisotropy Hamiltonian that we consider is $H_{\mathrm{MA}}=K \sin ^{2} \theta$, where $\theta$ is the angle that the sublattice magnetization makes with the anisotropy axis. $K$ is the anisotropy-energy density and can be written as the sum of two contributions, $K=K_{\text {mca }}+K_{\text {shape }}$. Furthermore, $K_{\text {mca }}$ is the energy density that corresponds to the magnetocrystalline anisotropy and $K_{\text {shape }}$ corresponds to the energy density for the shape anisotropy arising from the dipoledipole interaction. In our model, $K$ is independent of temperature and time, unlike what is proposed in Ref. [18]. The motivation for this choice is that the observed-temperature dependence of the magnetic anisotropy in such Fe-based systems is rather weak, as reported in Refs. [19,20], at least in the temperature interval relevant here. Although $K$ is the sum of both magneto-crystalline and shape-anisotropy contributions, we should point out that in our system the shape anisotropy should play much bigger role compared to its magneto-crystalline counterpart. One might expect a faster dynamics [21] if one considers a material with $K_{\text {mca }}>K_{\text {shape }}$; however, for a trilayer of $\mathrm{Fe} / \mathrm{Cr} / \mathrm{Fe}, K_{\mathrm{mca}}$ is typically not dominant. Furthermore, we choose $K$ in such a way that the hard axis is considered to be along the $z$ direction and the $x y$ plane is considered to be the easy plane, due to the shape anisotropy of thin-magnetic films and due to the possible intrinsic-uniaxial anisotropy. The last term of Eq. (2) is the interaction due to the applied-magnetic field. For the present case, it describes the pulsed field we apply during a short time of the simulations. Equation (1) is solved numerically using the procedure of Ref. [22].

For $T=0 \mathrm{~K}$, we consider the macrospin approximation, i.e., all atomistic spins within one layer are assumed to corotate. For finite-temperature simulations, one has to deal with this problem in an atomistic way, considering a large number of atoms in the simulations [12]. As mentioned, the exchange constants are calculated from first 


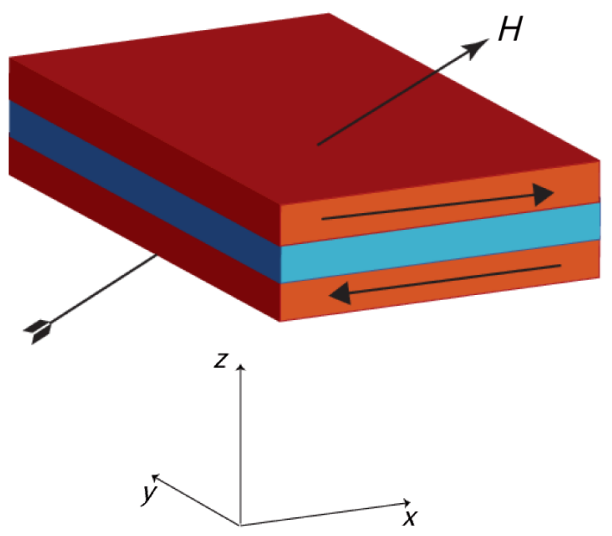

FIG. 1. The considered geometry of the atomistic simulations. The red and blue slabs represent $\mathrm{Fe}$ and $\mathrm{Cr}$, respectively. The arrows in the slabs represent directions of magnetization.

principles by mapping the ground-state-electronic structure to a Heisenberg Hamiltonian. The ground-stateelectronic structure is calculated using the Korringa-KohnRostocker Green's function method within the atomicsphere approximation [23,24]. In reality, the interlayer exchange between the Fe layers through the $\mathrm{Cr}$ spacer layer is influenced by the interface roughness of the $\mathrm{Fe}-\mathrm{Cr}$ interface, and to simulate this, we rescale our interlayer-exchange interactions with a factor of 10 , which is consistent with previous analyses $[25,26]$.

We consider that each Fe sublattice has a magnetization $\mathbf{M}=M_{s} \hat{\mathbf{n}}(t)$, where $\hat{\mathbf{n}}(t)$ is the unit vector. The magnitude of the effective-anisotropy field is given by $\mathbf{H}_{\mathrm{MA}}=$ $\frac{2 K}{M_{s}} \cos \theta \cdot M_{s}=\sum_{i} m_{s}^{i}$ is the saturation moment for the $\mathrm{Fe}$ sublattice. The initial value of a Fe-sublattice magnetization is along the positive or negative $x$ direction, as shown in Fig. 1, i.e., $\mathbf{M}= \pm M_{s} \hat{\mathbf{e}}_{\mathbf{x}}$. For all simulations, we use a square-shaped pulse of the external field. At time $t=0$, we apply a field of a constant value in the $x-z$ plane with a constant value described by $\mathbf{H}_{\mathbf{a}}=\left[H_{x}, 0, H_{z}\right]$. We study the cases where the magnetization was in plane, due to the shape anisotropy, without any further in-plane anisotropy.

\section{RESULTS}

We start by showing, in Fig. 2, an example of the magnetization dynamics of the trilayer shown in Fig. 1. Note that the magnetization shown in the figure is only for the top Fe slab in Fig. 1 (normalized to unity). The results shown are for a field pulse with $H_{x}=3 \mathrm{~T}$ and $H_{z}=$ $5 \mathrm{~T}$, for a duration of 5 picoseconds. The Gilbert damping constant used is 0.02 and the strength of the uniaxialanisotropy constant is $0.06 \mathrm{mRy} /$ atom, providing an easy-plane magnetization. At time $t=0$, we apply a field pulse. From the figure, we see that as soon as the pulse is applied, the magnetization is brought out of plane, and all the components of magnetizations are nonzero. The magnetization then precesses under the influence of

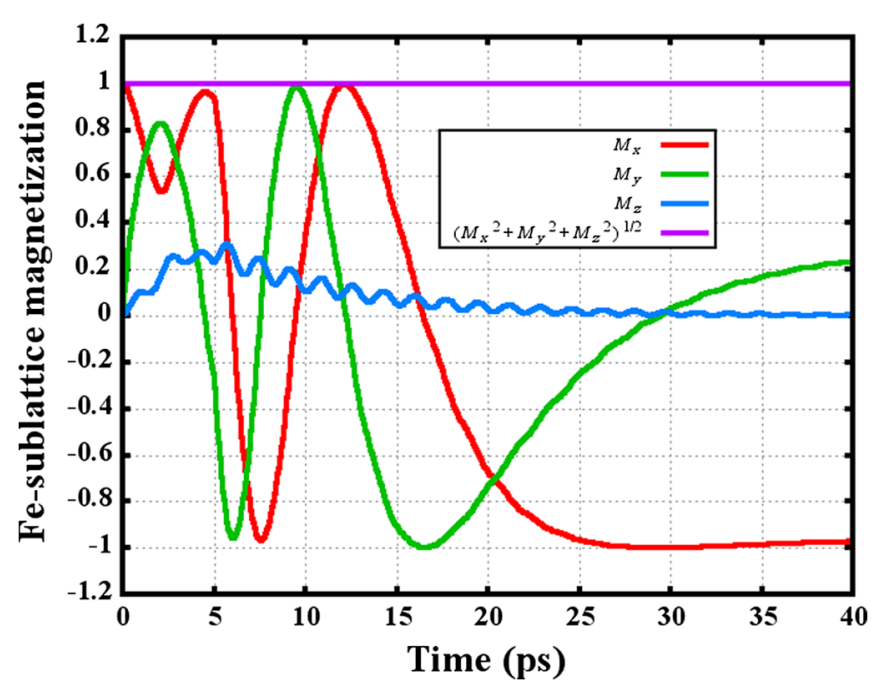

FIG. 2. The time evolution of the $x, y$, and $z$ components of the topmost $\mathrm{Fe}$-sublattice magnetization of the trilayer shown in Fig. 1. In addition, the magnitude of the magnetization is also shown. We have used $H_{x}=3 \mathrm{~T}, H_{z}=5 \mathrm{~T},|K|=0.06 \mathrm{mRy}$, and $\alpha=0.02$.

the external field, as well as the internal fields, provided by the anisotropy field and the exchange field due to the IEC. From the figure, it is clear that even after the external-magnetic field is switched off (i.e., after 5 ps) the system continues to evolve in time, and the magnetization dynamics continues over the entire time interval shown in the figure. At a sufficiently long simulation time, it seems, however, that the $x$ component of the magnetization approaches the negative $x$ direction. Hence, for this choice of external pulse, a switching of the magnetization occurs, and the direction of all magnetic moments illustrated in Fig. 1 has become reversed. The data in Fig. 2 are similar to those demonstrated experimentally in Ref. [10], in that the magnetization dynamics continues long after the external pulse has been first applied and then removed from the system.

By sweeping the strength of the $x$ component of the applied field, we can realize different situations, in which the sublattice magnetization of one of the Fe slabs in Fig. 1 rotates half a turn $\left(180^{\circ}\right)$, a whole turn $\left(360^{\circ}\right)$, or even more than one turn. In Fig. 3 we illustrate this result in a phase diagram of the switching behaviors. In the simulations shown in Fig. 3, we have used the same $z$ component of the applied field, the same Gilbert damping factor, pulsing time, and the same uniaxial-anisotropy constant, as used in Fig. 2. The figure only contains information about the Fe magnetization of the top Fe slab of Fig. 1. In the phase diagram of Fig. 3, the time of the simulations is shown on the $y$ axis and the magnitude of the $x$ component of the applied field is shown on the $x$ axis. The switched and unswitched regions are represented in terms of a color coding. The red color specifies that the magnetization has turned $n \cdot 360^{\circ}(n=0,1,2, \ldots)$, whereas the blue color 
(a) For $\alpha=0.02$

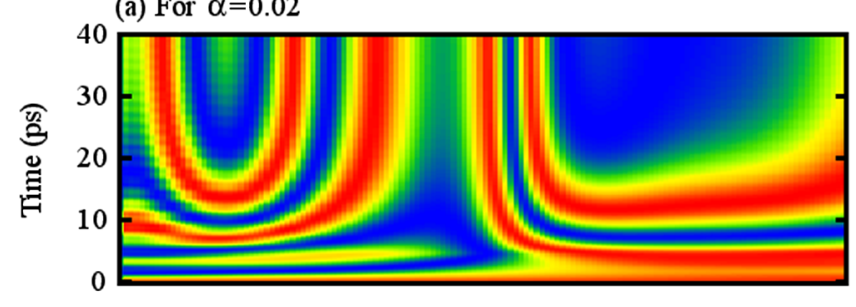

(b) For $\alpha=0.002$

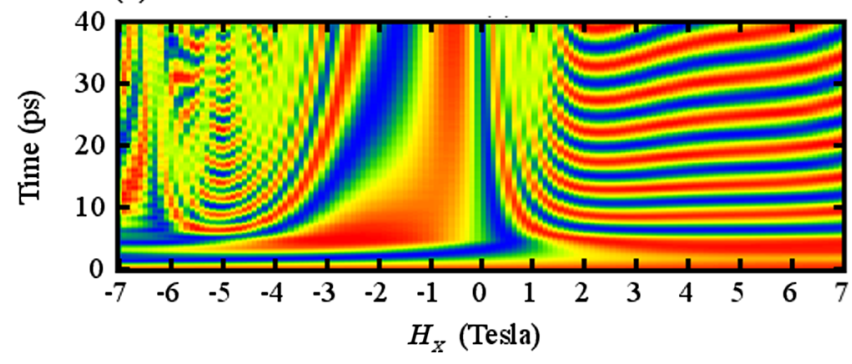

FIG. 3. The switching phase diagram of an $\mathrm{Fe} / \mathrm{Cr} / \mathrm{Fe}$ trilayer at $T=0 \mathrm{~K}$. The duration of the magnetic pulse is $5 \mathrm{ps}$, and the $x$ component of the magnetic field is shown on the $x$ axis, whereas the $z$ component is $5 \mathrm{~T}$. A uniaxial anisotropy of $0.06 \mathrm{mRy}$ is considered in the calculations, providing an easy-plane magnetization. The $x$ component of the magnetization of the top Fe slab of Fig. 1 is shown in a color scheme. The blue (red) areas indicate the regions where switching has occurred (not occurred). (Also, see the text for a description.) In (a) we show simulations for a damping parameter of 0.02 and in (b) the damping parameter is $0.002 . H_{z}=5 \mathrm{~T}$ and $|K|=0.06 \mathrm{mRy}$.

specifies that the magnetization has turned $n \cdot 360^{\circ}+$ $180^{\circ}(n=0,1,2, \ldots)$. Taking $H_{x}=5 \mathrm{~T}$ as an example, Fig. 3(a) shows that when the pulse arrives, the magnetization of the top Fe slab is along the positive $x$ direction (red color), but after 5-6 ps the magnetization has reversed to the negative $x$ direction, and after $10 \mathrm{ps,} \mathrm{a} \mathrm{rotation} \mathrm{of}$ $360^{\circ}$ to the positive $x$ direction has occurred. In between, the magnetization, of course, points along the positive or negative $y$ direction (this component is not shown), and hence exhibits a rotational behavior. After some $30 \mathrm{ps}$, the magnetization finally settles down along the negative $x$ direction. Overall, the data in Fig. 3 show that the magnetization dynamics continues long after the external pulse has been turned off, in a similar fashion as observed experimentally [10].

Another interesting feature of the data in Fig. 3(a) is that the results are not symmetric with respect to the positive or negative $x$ component to the applied field. At first sight, this might seem puzzling, since the geometry shown in Fig. 1 is very symmetric with respect to the positive and negative $x$ directions. However, the data in Fig. 3(a) depict only the magnetization of the topmost Fe layer, and for this layer alone there is no obvious symmetry for the cases when the applied pulse is along the positive or negative $x$ direction, and the patterns in Fig. 3(a) for positive and negative values of $H_{x}$ are different. However, for all values of $H_{x}$, we find that after a sufficiently long time the magnetization has settled down into a given orientation.

There are two torques that, in general, are relevant for the magnetization dynamics, a precessional torque and a damping torque, and the influence of the latter is provided in the simulations by the strength of the Gilbert damping parameter. Hence, one may expect that the phase diagram should depend critically on this parameter and, to investigate this possibility, we show, in Fig. 3(b), a simulation with all parameters kept exactly the same as for the simulation shown in Fig. 3(a); the one difference is that the Gilbert damping parameter is set to 0.002, i.e., 10 times lower than that used for Fig. 3(a). In this case, the rotation of the magnetization continues throughout the entire period of the simulations, at least for positive values of $H_{x}$, which shows that it is the damping parameter that primarily determines when the magnetization stops rotating.

As the pulse hits the sample (simulation box), the angle between the two Fe slabs starts to deviate from the $180^{\circ}$ antiferromagnetic coupling. To illustrate this fact, we show, in Fig. 4, a contour plot for the angle between two Fe-sublattice magnetizations, in which red coloration signifies a $180^{\circ}$ antiferromagnetic coupling. This plot corresponds to the simulations shown in Figs. 3(a) and 3(b). As is clear from Figs. 4(a) and 4(b), the magnetization of the two Fe sublattices is initially at $180^{\circ}$ with each other, but after the external field is switched on and time evolves, the angle between the sublattices is reduced from $180^{\circ}$ and oscillates with time, especially when $H_{x} \neq 0$. Figures 4(a) and 4(b) show data for two different damping parameters, and we note that these two different damping parameters result in slightly different magnetization dynamics, in that it takes longer for the angle between the two sublattices to approach $180^{\circ}$ for the weakly damped system. However,

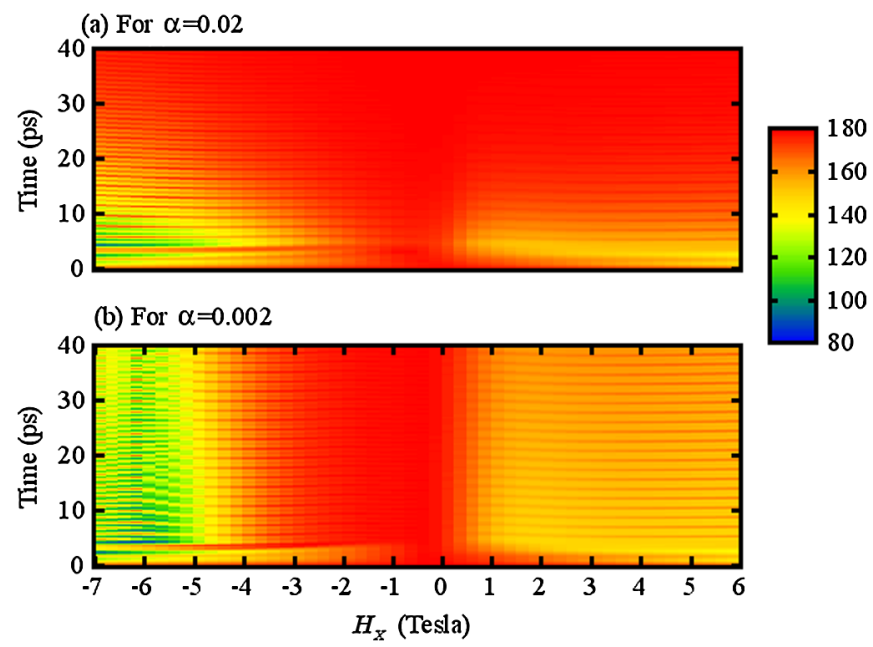

FIG. 4. Evolution of the angle between the sublattice magnetizations: (a) for a damping factor of 0.02 , and (b) with a damping factor of 0.002 . In the simulations, the $x$ component to the applied field was varied, whereas the $z$ component was kept constant, $H_{z}=5 \mathrm{~T}$, also $|K|=0.06 \mathrm{mRy}$. 
for both simulations a $180^{\circ}$ coupling is obtained when $t \rightarrow \infty$. The minimum angle is seen when the magnitude of the $x$ component of the applied field is largest, after $5 \mathrm{ps}$ (i.e., the duration of the pulse), where the two sublattice magnetizations are approximately perpendicular to each other. This means that, with the applied field as a driving force, the angle between the two sublattices is increasingly deviating, albeit in an oscillating fashion, from the antiferromagnetic coupling. When the angle between two sublattices is less than $180^{\circ}$ an internal-exchange field is built up in each $\mathrm{Fe}$ slab, which causes a dynamical response. In addition, a nonzero $z$ component to the magnetization (e.g., as shown in Fig. 2) builds up an anisotropy field. These two contributions to the effective field, which drives the magnetization dynamics, are nonzero long after the external field has been switched off. In fact, they are zero only after the magnetization has settled down to a constant value. Hence, the effective field is, in this case, dynamic, and the torques due to this field have, as usual, two components: the damping part and the precessional term. Another note on Fig. 4 is that, since the effective field is dynamic and changes with time, where the strength of the effective field decreases once the external field has been switched off, the period of the oscillations in Fig. 2 is time dependent.

The data in Fig. 4 demonstrate an important result, namely, that, during the short time the external field is applied, a significant energy is established both in the exchange interaction term $\mathcal{H}_{\text {ex }}$ as well as in the anisotropy energy $\mathcal{H}_{\mathrm{MA}}$ of Eq. (2). In a way, one can view the whole process as a result of the energy provided by the $\mathcal{H}_{a}$ term of Eq. (2) that, during the pulse duration, pumps energy into the other two terms, $\mathcal{H}_{\mathrm{ex}}$ and $\mathcal{H}_{\mathrm{MA}}$. Even if $\mathcal{H}_{a}$ is nonzero only for a short time, the other two terms will be nonzero for a much longer period, and the effective field composed of contributions, which are due to these two terms depending on $\mathbf{m}_{i}(t)$, carries some sort of information of the field pulse applied at an earlier stage.

To demonstrate this in a more formal way, we plot, in Fig. 5, the temporal evolution of the different components of magnetic energy during one of the simulations. From Fig. 5, one can see that the Zeeman energy is negative during the duration of the pulse whereas the exchange and anisotropy energy are positive. The sum of the three components, i.e., the total energy is negative during the duration of the pulse, which means that during the time that the pulse is applied, the energy is lowered and the system tries to relax into a new ground-state configuration. When the external pulse is switched off, Fig. 5 shows that there is still a positive energy in the exchange and the anisotropy contribution to the energy and the total energy is now positive. In this configuration, there is both a $z$ component of the magnetization as well as a noncollinear coupling between the moments in the two Fe slabs. The system then relaxes back to the original configuration of a

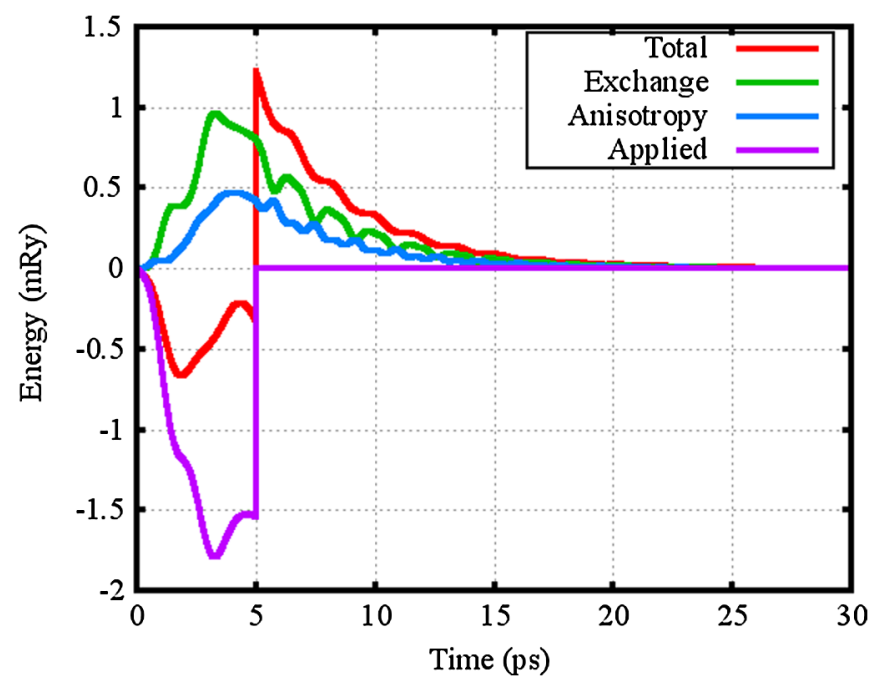

FIG. 5. Time evolution of different components of magnetic energy (exchange, anisotropy, applied), plotted along with the total magnetic energy. In this simulation the $x$ component to the applied field was $3 \mathrm{~T}$. Furthermore, $\mathrm{H}_{z}=5 \mathrm{~T}$ and $\alpha=0.02$.

collinear-antiferromagnetic coupling of the Fe slabs without a $z$ component to the magnetization, and it does this in the anisotropy and exchange field that has been built up during the duration of the external field. The relaxation back to the collinear configuration takes considerably longer than the duration of the field, as Fig. 5 shows. From Fig. 5, it can be seen that the energy due to the applied field (Zeeman energy) acts as a reservoir for the other two terms (exchange and anisotropy) of the magnetic Hamiltonian. Hence, in order to analyze the magnetization dynamics provided in Figs. 2 and 3, one does not need to invoke concepts like the inertia of antiferromagnetic unit vectors [10]. We suggest a simpler interpretation based on a redistribution of energy among the three terms in Eq. (2), in particular, a redistribution of energy from $\mathcal{H}_{a}$ to $\mathcal{H}_{\text {ex }}$ and $\mathcal{H}_{\mathrm{MA}}$, during the time period in which the external field is applied. This does not mean that the analysis of Ref. [10], building on antiferromagnetic unit vectors, is wrong. We simply propose that the phenomenon observed in Ref. [10] is possible to observe in more general situations, also in ferromagnetic materials. A way to analyze these more general situations is the dynamic redistribution of energy from the Zeeman energy to any of the other terms of the spin Hamiltonian.

The analysis in the preceding paragraph indicates that the strength of the anisotropy also influences the magnetization dynamics and to investigate this further we plot, in Fig. 6, the simulated data as a function of the uniaxialanisotropy constant $K$. All other parameters are the same as those for the simulation of Fig. 3(a). First, we note that when $K=0$, the tendency of switching is reduced, since in this case the dynamics is provided by the exchange field alone, via the IEC. However, when $K$ increases to 0.01-0.04 mRy, the tendency for switching increases and 


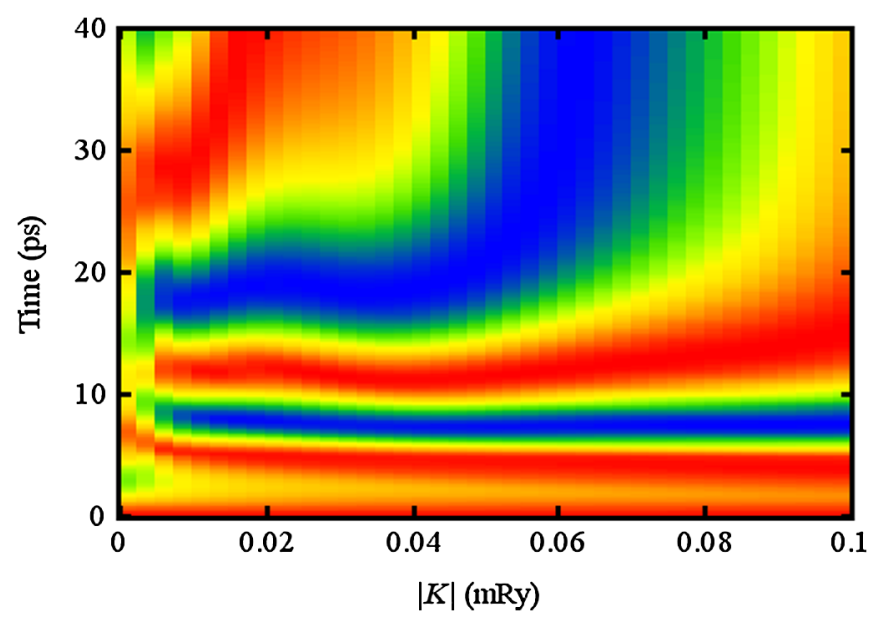

FIG. 6. Switching times as a function of out-of-plane anisotropy. The duration of the magnetic pulse is $5 \mathrm{ps}$, the $z$ component of the applied magnetic field is $5 \mathrm{~T}$, and the $x$ component is $3 \mathrm{~T}$. (All other parameters are the same as Fig. 2.)

the magnetization is seen to precess twice before stabilizing along the positive $x$ direction. For even larger values of anisotropy energy, $K$ is larger than $\approx 0.05 \mathrm{mRy}$, and only one revolution is found in the simulations, before the magnetization is stabilized. Hence, there is a critical value of $K$ that provides an optimally large amount of revolutions in the magnetization.

We also investigate the case when the interlayerexchange coupling is zero, in Eq. (2), by performing simulations only to the topmost Fe slab of Fig. 1, i.e., a purely ferromagnetic system having an easy-plane anisotropy. The simulated results are shown in Fig. 7, and we note that in this case the magnetization also behaves as if it had inertia, i.e., it proceeds after an external field is

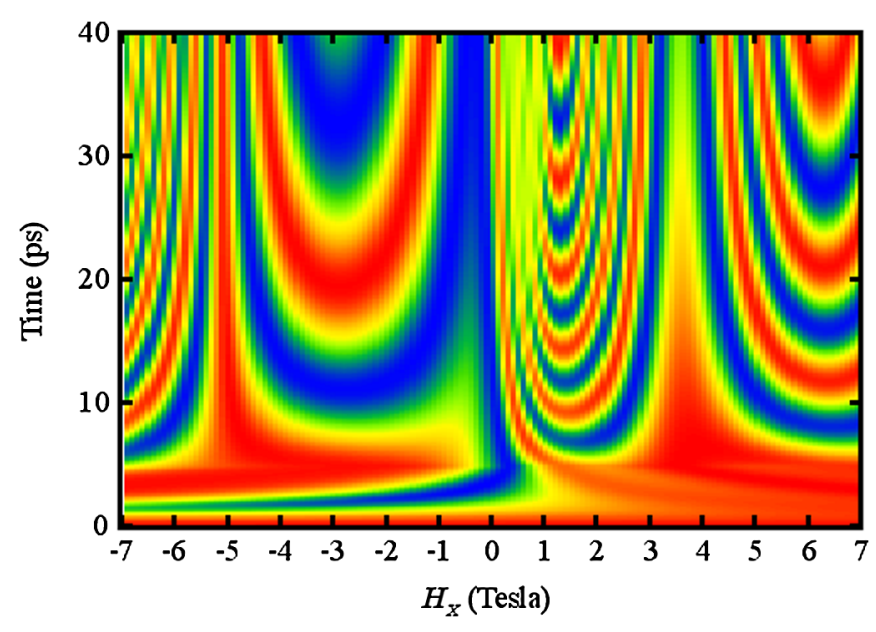

FIG. 7. Switching-phase diagram for $\mathrm{Fe}_{3}$ layers with the same set of parameters as indicated in Fig. 3 for the $\mathrm{Fe}_{3} / \mathrm{Cr}_{4} / \mathrm{Fe}_{3}$ trilayer, except for the IEC, which was set to be equal to zero. In these simulations, the $x$ component to the applied field was varied, whereas the $z$ component was kept constant, $H_{z}=5 \mathrm{~T}$, $|K|=0.06 \mathrm{mRy}$, and $\alpha=0.02$. switched off. An analysis relying on antiferromagnetic unit vectors becomes cumbersome in this case, but the pumping of energy from $\mathcal{H}_{a}$ to the two other components given in Eq. (2), in this case the anisotropy energy, provides an easy interpretation. The data in Fig. 7, are actually consistent with the experiments performed in Ref. [27], where a short pulse was applied to a ferromagnet thin film.

\section{CONCLUSIONS}

In conclusion, we have performed atomistic spindynamics simulations for a synthetic antiferromagnet subjected to a short-magnetic pulse. We have demonstrated that the magnetization dynamics continues long after the pulse has been applied and removed, in a fashion that is consistent with the experimental results of Ref. [10].

Several materials parameters, such as the damping and strength of the magnetic anisotropy, are found to influence the details of the magnetization dynamics. We suggest that such magnetization dynamics could be understood in terms of a redistribution of energy, during the time that the pulse is applied, so that the magnetic-anisotropy energy and interlayer-exchange energy are out of equilibrium. The magnetic energy due to the incident pulse, hence, becomes distributed dynamically to the effective interlayerexchange coupling between the Fe layers, and it also modifies the magnetic-anisotropy energy. The latter term ensures that the observed behavior of a magnetization dynamics, which progresses long after an external field has been applied and switched off, is also possible in ferromagnetic materials.

Our suggestion for why the magnetization dynamics persists long after any external field is switched off is that the initial pulse brings the system to a new intermediate state, in which each atomistic spin precesses in a local field composed of the applied field and an anisotropy and exchange field, which is built up when the external field is applied. After the external field is removed, each atomistic spin continues to precess in the anisotropy and exchange field and subsequently, a new final state is realized.

The interaction between the atomistic spins and the relevant fields (applied, exchange, and anisotropy) is, despite the seemingly simple first-order form of Eq. (1), quite a complex interaction in which higher-order nonlinear couplings play an important role. This is due to the nonlocal nature of the exchange interactions, where each atomistic spin interacts with all other spins via an interatomic exchange, and this interaction sometimes competes with the anisotropy energy and the Zeeman energy from the applied field. Hence, the time evolution of the ensemble of atomistic spins represents a highly nonlinear phenomenon, which carries over to a nonlinear evolution of $\mathcal{H}_{\mathrm{MA}}$ and $\mathcal{H}_{\text {ex }}$ in Eq. (2).

We illustrate this by considering a simple example, in which, for simplicity, we consider only the precession term 
without thermal fluctuations. It is then easy to show from Eq. (1) that the acceleration of the precession of any selected atomic spin $\frac{d^{2} \mathbf{m}_{i}}{d t^{2}}$, is related to a balance between the precession rate of all other spins and the time evolution of the applied and anisotropy fields, i.e., $\frac{d^{2} \mathbf{m}_{i}}{d t^{2}}=\gamma \mathbf{m}_{i} \times$ $\sum_{j}\left(J_{i j} \frac{d \mathbf{m}_{j}}{d t}+\frac{\mathbf{H}_{\mathrm{MA}}}{d t}+\frac{\mathbf{H}_{\mathbf{a}}}{d t}\right)$, where $\mathbf{H}_{\mathrm{MA}}$ and $\mathbf{H}_{\mathrm{a}}$ are the contributions to the effective field from the magnetic anisotropy and the applied field, respectively. From this expression it is clear that there is a finite acceleration of the spins as long as the system is out of equilibrium even in the absence of an external field. In particular, the contribution from the magneto-crystalline anisotropy can give rise to a finite acceleration even for a perfectly collinear-ferromagnetic system. By a consideration of the damping term and finitetemperature effects, higher-order terms also enter.

In the present work, we have chosen to show only results from simulations at $T=0 \mathrm{~K}$, although finite-temperature simulations give a similar overall picture (data not shown). At elevated temperatures, effects that lie outside the present treatment also become important, although they do not destroy the dynamical evolution of the effective field, as is discussed here. These finite-temperature effects involve the excitation of Stoner pairs and the thermalization of the atomistic spins [28]. Such effects can be at least parametrized in simulations of the kind performed here, but this is outside the scope of the present study.

\section{ACKNOWLEDGMENTS}

We are grateful to the Swedish Research Council for their support. O.E. also acknowledges support from the KAW Foundation and the ERC (Grant No. 247062-ASD). A. B. acknowledges eSSENCE. The simulations were performed on resources provided by the Swedish National Infrastructure for Computing (SNIC) at the National Supercomputer Centre (NSC). Valuable discussions with Professor J. Stöhr, Professor A. Kirilyuk, and the late Professor H.-C. Siegmann are acknowledged.

[1] E. Beaurepaire, J.-C. Merle, A. Daunois, and J.-Y. Bigot, Ultrafast Spin Dynamics in Ferromagnetic Nickel, Phys. Rev. Lett. 76, 4250 (1996).

[2] Y. Zheng et al., Magnetic Random Access Memory (MRAM), J. Nanosci. Nanotechnol. 7, 117 (2007).

[3] M. N. Baibich, J. M. Broto, A. Fert, F. Nguyen Van Dau, F. Petroff, P. Etienne, G. Creuzet, A. Friederich, and J. Chazelas, Giant Magnetoresistance of (001)Fe/(001)Cr Magnetic Superlattices, Phys. Rev. Lett. 61, 2472 (1988).

[4] G. Binasch, P. Grünberg, F. Saurenbach, and W. Zinn, Enhanced Magnetoresistance in Layered Magnetic Structures with Antiferromagnetic Interlayer Exchange, Phys. Rev. B 39, 4828 (1989).

[5] W.H. Butler, X.-G. Zhang, T.C. Schulthess, and J.M. MacLaren, Spin-Dependent Tunneling Conductance of FelMgOlFe Sandwiches, Phys. Rev. B 63, 054416 (2001).
[6] J. S. Moodera, L. R. Kinder, T. M. Wong, and R. Meservey, Large Magnetoresistance at Room Temperature in Ferromagnetic Thin Film Tunnel Junctions, Phys. Rev. Lett. 74, 3273 (1995).

[7] J. Åkerman, Toward a Universal Memory, Science 308, 508 (2005).

[8] C. D. Stanciu, F. Hansteen, A. V. Kimel, A. Kirilyuk, A. Tsukamoto, A. Itoh, and Th. Rasing, All-Optical Magnetic Recording with Circularly Polarized Light, Phys. Rev. Lett. 99, 047601 (2007).

[9] A. Bergman, B. Skubic, J. Hellsvik, L. Nordström, A. Delin, and O. Eriksson, Ultrafast Switching in a Synthetic Antiferromagnetic Magnetic Random-Access Memory Device, Phys. Rev. B 83, 224429 (2011).

[10] A. V. Kimel, B. A. Ivanov, R. V. Pisarev, P. A. Usachev, A. Kirilyuk, and Th. Rasing, Inertia-Driven Spin Switching in Antiferromagnets, Nature Phys. 5, 727 (2009).

[11] V.P. Antropov, M. I. Katsnelson, B. N. Harmon, M. van Schilfgaarde, and D. Kusnezov, Spin Dynamics in Magnets: Equation of Motion and Finite Temperature Effects, Phys. Rev. B 54, 1019 (1996).

[12] B. Skubic, J. Hellsvik, L. Nordström, and O. Eriksson, A Method for Atomistic Spin Dynamics Simulations: Implementation and Examples, J. Phys. Condens. Matter 20, 315203 (2008).

[13] A. I. Liechtenstein, M. I. Katnelson, and V. A. Gubanov, Exchange Interactions and Spin-Wave Stiffness in Ferromagnetic Metals, J. Phys. F 14, L125 (1984); Local Spin Density Functional Approach to the Theory of Exchange Interactions in Ferromagnetic Metals and Alloys, J. Magn. Magn. Mater. 67, 65 (1987).

[14] J. Miltat, G. Albuquerque, and A. Thiavill, Spin Dynamics in Confined Magnetic Structures (Springer-Verlag, Berlin, 2001).

[15] Y. Tserkovnyak, A. Brataas, G.E.W. Bauer, and B.I. Halperin, Nonlocal Magnetization Dynamics in Ferromagnetic Heterostructures, Rev. Mod. Phys. 77, 1375 (2005).

[16] E. Runge and E. K. U. Gross, Density-Functional Theory for Time-Dependent Systems, Phys. Rev. Lett. 52, 997 (1984).

[17] K. Blum, Density Matrix Theory and Applications (Plenum press, New York, 2010).

[18] J.-Y. Bigot, M. Vomir, L.H.F. Andrade, and E. Beaurepaire, Ultrafast Magnetization Dynamics in Ferromagnetic Cobalt: The Role of the Anisotropy, Chem. Phys. 318, 137 (2005).

[19] J. B. Staunton, S. Ostanin, S. S. A. Razee, B. L. Gyorffy, L. Szunyogh, B. Ginatempo, and E. Bruno, Temperature Dependent Magnetic Anisotropy in Metallic Magnets from an $A b$ Initio Electronic Structure Theory: L1 $1_{0}$-Ordered FePt, Phys. Rev. Lett. 93, 257204 (2004).

[20] P. Asselin, R. F. L. Evans, J. Barker, R. W. Chantrell, R. Yanes, O. Chubykalo-Fesenko, D. Hinzke, and U. Nowak, Constrained Monte Carlo Method and Calculation of the Temperature Dependence of Magnetic Anisotropy, Phys. Rev. B 82, 054415 (2010).

[21] J.-Y. Bigot, M. Vomir, and E. Beaurepaire, Coherent Ultrafast Magnetism Induced by Femtosecond Laser Pulses, Nature Phys. 5, 515 (2009). 
[22] J. H. Mentink, M. V. Tretyakov, A Fasolino, M. I Katsnelson, and Th. Rasing, Stable and Fast Semi-implicit Integration of the Stochastic Landau-Lifshitz, Equation, J. Phys. Condens. Matter 22, 176001 (2010).

[23] J. Korringa, On the Calculation of the Energy of a Bloch Wave in a Metal, Physica (Amsterdam) 13, 392 (1947).

[24] W. Kohn and N. Rostoker, Solution of the Schrdinger Equation in Periodic Lattices with an Application to Metallic Lithium, Phys. Rev. 94, 1111 (1954).

[25] J. Kudrnovsky, V. Drchal, I. Turek, M. Sob, and P. Weinberger, Interlayer Magnetic Coupling: Effect of Interface Roughness, Phys. Rev. B 53, 5125 (1996).
[26] E. Holmström, L. Bergqvist, B. Skubic, B. Hjörvarsson, L. Nordström, I. Abrikosov, P. Svedlindh, and O. Eriksson, On the Sharpness of the Interfaces in Metallic Multilayers, Proc. Natl. Acad. Sci. U.S.A. 101, 4742 (2004).

[27] C. Stamm, I. Tudosa, H. C. Siegmann, J. Stöhr, A. Yu. Dobin, G. Woltersdorf, B. Heinrich, and A. Vaterlaus, Dissipation of Spin Angular Momentum in Magnetic Switching, Phys. Rev. Lett. 94, 197603 (2005).

[28] L. Guidoni, E. Beaurepaire, and J.-Y. Bigot, Magnetooptics in the Ultrafast Regime: Thermalization of Spin Populations in Ferromagnetic Films, Phys. Rev. Lett. 89, 017401 (2002). 